\title{
Rotational Paper-Based Microfluidic-Chip Device for Multiplexed and Simultaneous Fluorescence Detection of Phenolic Pollutants Based on a Molecular-Imprinting Technique
}

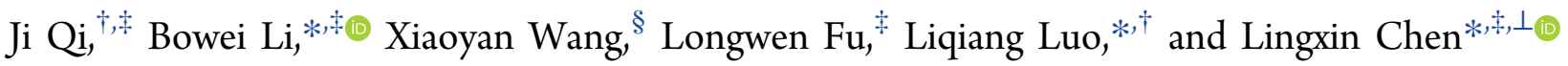 \\ ${ }^{\dagger}$ College of Sciences, Shanghai University, Shanghai 200444, China \\ ${ }^{\ddagger}$ CAS Key Laboratory of Coastal Environmental Processes and Ecological Remediation, Yantai Institute of Coastal Zone Research, \\ Chinese Academy of Sciences, Yantai 264003, China \\ ${ }^{\S}$ School of Pharmacy, Binzhou Medical University, Yantai 264003, China \\ ${ }^{\perp}$ College of Chemistry and Chemical Engineering, Qufu Normal University, Qufu 273165, China
}

Supporting Information

ABSTRACT: In this study, we first present rotational paper-based microfluidic chips (RPADs) combined with a molecular-imprinting (MIP) technique to detect phenolic pollutants. The proposed rotational paper-based microfluidic chips could implement qualitative and quantitative analysis of two different phenolic contaminants, 4nitrophenol (4-NP) and 2,4,6-trinitrophenol (TNP), simultaneously. Qualitative and quantitative analysis could be implemented simultaneously through fluorescence-intensity changes depending on the structures of quantum dots combined with a molecular-imprinting technique. Moreover, the rotational paper-based microfluidic chips provide a low cost, flexible, and easy way to operate the entire process conveniently. Under the optimal conditions, the proposed sensors showed high sensitivity and selectivity. Our final experimental results illustrated that the detection limits of 4-NP and TNP in the paper-based quantum-dot MIP (PQ-MIP) RPADs ranged from 0.5 to $20.0 \mathrm{mg} / \mathrm{L}$, with detection limits of 0.097 and $0.071 \mathrm{mg} / \mathrm{L}$, respectively. This novel rotational paper-based microfluidic device shows great potential and versatility for multiplexed, portable, and rapid testing of environmental and biological samples in the future.

$\mathrm{M}$ icrofluidic paper-based analytical devices ( $\mu$ PADs) have attracted considerable attention ${ }^{1,2}$ owing to their advantages of convenience, low cost, simple fabrication, small size. As a class of devices, common $\mu$ PADs are limited to assays because of the difficulty of finding a paper-based mechanism that can be easily operated. ${ }^{3}$ Liu and Crooks first introduced $3 \mathrm{D}$ origami-based paper analytical devices (3D oPADs) and proved their usability for simple glucose and protein analysis. ${ }^{4}$ Because of their strong operability, 3D paper chips have been widely researched. ${ }^{5}$ Currently, $\mu$ PADs have been combined with various detection techniques for chemical analysis, ${ }^{6}$ such as colorimetry, ${ }^{7,8}$ luminescence, ${ }^{9}$ electrochemical detection, ${ }^{10,11}$ surface-enhanced Raman scattering, ${ }^{2}$ and fluorescence. $^{12}$ The proper integration of these techniques would address the compatibility between the chip and the analyzing instruments during the laboratory analysis and would be favorable for performing sample-to-result tests in a simple, fast, and cost-effective way. ${ }^{13}$ Marta et al. demonstrated that microfluidic paper-based analytical devices were compatible with simple instrumental equipment based on the extremely cheap photometric and fluorometric paired-emitter-detectordiode (PEDD) detectors. ${ }^{14}$ Guzman et al. reported a $\mu \mathrm{PAD}$ combined with a portable detection instrument for lowconcentration-formaldehyde detection. ${ }^{15} \mathrm{Yu}$ et al. demonstrated a functionalized 3D paper-based device for point-of-care diagnosis by electrochemical immunoassays, which allowed the chip to work well with electrochemical instruments. ${ }^{16} \mathrm{~A}$ highthroughput sample-inspection design was also an important manifestation of the flexibility of paper chips. Martinez et al. first demonstrated the stacked design for $3 \mathrm{D} \mu \mathrm{PADs}$ with layers of paper; this device had a top layer with four inputs stacked on a bottom layer and an array of 1024 detection spots. ${ }^{17}$ In a previous work, we designed a movable valve on paper-based microfluidic devices for manipulating capillarydriven fluids, ${ }^{3}$ and rotary microfluidic PMMA-based chips have been reported. ${ }^{18}$ In this work, we further developed the rotational paper chip for fluorescence analysis. The rotational paper chip can be very compatible with fluorescence instruments. In addition, this work enabled the multiplexed detection of multiple MIP targets.

Received: March 22, 2018

Accepted: August 23, 2018

Published: August 23, 2018 
Environmental pollution can undermine the safety of water quality and increase the probability of livestock and human exposure to waterborne diseases. ${ }^{19}$ With the advantages of simplicity of operation, good flexibility, and portability, microfluidic analytical devices may potentially be a powerful platform for environmental monitoring. ${ }^{20,21}$ The extensive use of nitrophenols has caused the production of refractory toxic organic pollutants in industrial wastewater. ${ }^{22,23}$ Among nitrophenol contaminants, 4-nitrophenol (4-NP) and 2,4,6trinitrophenol (TNP) are common. The allowable level for TNP in drinking water is set at $0.5 \mathrm{mg} / \mathrm{L} .{ }^{24}$ Alkasir et al. used functional materials deposited on filter paper and constructed a colorimetric bioassay for the detection of phenolic compounds. ${ }^{25}$ Yan et al. developed a photoelectrochemical sensor for detecting pentachlorophenol by combined surface-imprinting technology and paper-based microfluidic-chip techniques. $^{26}$ Kong et al. reported a microfluidic paper-based colorimetric sensor based on molecularly imprinted polymer (MIPs) membranes for the detection of bisphenol A. ${ }^{27}$ Liu et al. reported Mn-doped $\mathrm{ZnS}$ quantum dots (QDs) capped by molecularly imprinted polymers for the chemiluminescence detection of 4-nitrophenol in tap water. ${ }^{28}$ Because of the MIP property of high selectivity, the made sensors can detect substances in complicated environments. ${ }^{29,30}$ Moreover, because of the high sensitivity, excellent specificity, and simplicity of the detection mechanism, fluorescence-based molecularly imprinted polymer (FL-MIP) detection was applied to paper-based sensors recently. ${ }^{31-34}$

To the best of our knowledge, this is the first demonstration to combine rotational paper-based microfluidic chips with the MIP technique to realize rapid and multiplexed detection, and this platform can qualitatively and quantitatively analyze different environmental pollutants with good selectivity and performance. Because of the paper valve's rotation mechanism, one can effectively use space and achieve multiplexed detection on the chip. In addition, 4-nitrophenol (4-NP) and 2,4,6trinitrophenol (TNP) samples were successfully analyzed on the $\mu$ PADs. The convenient fabrication and flexible operation of the rotational valves could make it convenient for untrained users to conduct qualitative and quantitative detection of environmental pollutants. This new paper-based MIP platform may open up a new avenue for the investigation of the fluorescence effects of MIP reactions in environmental analysis.

\section{EXPERIMENTAL SECTION}

Materials. Some raw chemicals, such as sodium $\mathrm{N}$ hydroxysuccinimide (NHS), 3-ethylcarbodiimide hydrochloride (EDC), 3-aminopropyltriethoxysilane (APTES), borohydride $\left(\mathrm{NaBH}_{4}\right)$, and cadmium chloride $\left(2 \mathrm{CdCl}_{2} \cdot 5 \mathrm{H}_{2} \mathrm{O}\right)$, were purchased from Aladdin. 2-N-Morpholinoethanesulfonic acid (MES) was purchased from Sigma-Aldrich. In addition, most raw chemicals, such as tellurium powder, tetramethoxysilane (TEOS), ammonia solution $\left(\mathrm{NH}_{3} \cdot \mathrm{H}_{2} \mathrm{O}\right)$, thioglycolic acid (TGA), L-cysteine (L-Cys), glutathione (GSH), 4-nitrophenol (4-NP), 2,4,6-trinitrophenol (TNP) ethanol, and other affiliated chemicals, were obtained from Sinopharm Chemical Reagent Company Ltd. All solvents and chemicals were of pure, analytical grade. The Pall Cascada laboratory water system (Millipore) produced ultrapure water (18.2 M $\Omega$ ). Glass-fiber paper was obtained from Blue Sky Environmental Monitoring Products Factory.

Instrumentation. The $\mu \mathrm{PADs}$ were designed by drawing software (Adobe Illustrator). Whatman chromatography paper
(Whatman chromatography No.1 paper, GE) was printed on a XEROX Phaser 8560DN wax inkjet printer with a maximum resolution of $2400 \mathrm{dpi}$. The morphology of the sensing site was researched by scanning electron microscopy (SEM, JSM 5600 LV). A spectrofluometer (Fluoromax-4, HORIBA) was used to obtain fluorescence spectra. The excitation wavelength was 396 $\mathrm{nm}$, and the emission spectra were scanned from 450 to 650 $\mathrm{nm}$ for the quantum dots. UV-vis absorption spectra were recorded with a spectrophotometer (NanoDrop 2000/2000 C, Thermo Scientific) to research the elution template process of the molecularly imprinted polymers. In addition, a fluorescence microscope (IX51, Olympus) equipped with a CCD camera was used to obtain fluorescence-microscopy photographs.

Synthesis of Fluorescence-Based Carboxyl-Terminated QDs. The preparation of quantum dots followed that of our previous study. ${ }^{35}$ In the synthesis process, mercaptoacetic acid, cysteine, and glutathione were added to modify the CdTe QDs.

Synthesis of Glass-Paper-Based FL-MIP Sensors. The synthetic methods was those previously reported, ${ }^{33}$ and the amount of each component and details were improved. The glass-fiber paper was $5 \times 5 \mathrm{~mm}$; it was immersed in a $0.2 \mathrm{~mol} /$ $\mathrm{L} \mathrm{HCl}$ solution for $30 \mathrm{~min}$ and then washed twice with DI water to wash off excess hydrochloric acid. The pieces were then soaked in $20 \mathrm{~mL}$ of a $50 \%$ ethanol solution, and $200 \mu \mathrm{L}$ of APTES was added. The pieces were incubated for $4 \mathrm{~h}$ and then washed three times with DI water to remove the excess ethanol. The slide was mounted on a Petri dish with a glass slide to prevent random floating. CdTe QDs $(7.5 \mathrm{~mL})$ were added, followed by $3 \mathrm{~mL}$ of $20 \mathrm{mg} / \mathrm{mL}$ EDC and $3 \mathrm{~mL}$ of 10 $\mathrm{mg} / \mathrm{mL}$ NHS ( $\mathrm{pH}$ 5.2). After being shaken at room temperature for $12 \mathrm{~h}$, the paper was grafted with quantum dots, and then it emitted a uniform green light when irradiated with UV light. Subsequently, the paper grafted with quantum dots was further wrapped with an MIP layer. First, functional monomers (APTES) and two template molecules (4-NP and TNP) were separately mixed and prepolymerized. The reaction was prepolymerized with 130-150 rpm shaking for a suitable amount of time (the prepolymerization time of 4-NP was 30 $\mathrm{min}$, and that of TNP was $1 \mathrm{~h}$ ). Then, the appropriate amounts of $\mathrm{NH}_{3} \cdot \mathrm{H}_{2} \mathrm{O}$ and TEOS were added simultaneously. In the event of white turbidity, the paper grafted with quantum dots was placed in the dark at room temperature overnight and shaken at 130-150 rpm. Finally, the paper containing the molecularly imprinted layer was eluted to remove the template molecule by using the corresponding eluent. During the whole process, the fluorescent molecularly imprinted grating on the paper was successfully fabricated (PQ-4-NP-MIP and PQTNP-MIP).

The procedure for synthesizing 4-NP-MIP was as follows: 70 $\mu \mathrm{L}$ of functional monomer APTES, $70 \mu \mathrm{L}$ of TEOS, $70 \mu \mathrm{L}$ of initiator $\mathrm{NH}_{3} \cdot \mathrm{H}_{2} \mathrm{O}$, and $4 \mathrm{mg}$ of $4-\mathrm{NP}$ were added together. For the TNP-MIP synthesis process, the template molecule, TNP (5 mg); functional monomer, APTES (80 $\mu \mathrm{L})$; crosslinking agent, TEOS $(80 \mu \mathrm{L})$; and initiator, $\mathrm{NH}_{3} \cdot \mathrm{H}_{2} \mathrm{O}(70$ $\mu \mathrm{L}$ ), were added. Another group without a template (neither 4NP nor TNP) was recorded as NIP as a control. Finally, the products were shocked and washed with ethanol/acetonitrile $(8: 2, \mathrm{v} / \mathrm{v})$ to remove the templates. The PQ-4-NP-MIP and PQ-TNP-MIP paper chips were saved at $4{ }^{\circ} \mathrm{C}$ in the dark until use.

Fabrication and Assembling of RPADs. The paperbased-quantum-dot-MIP-RPAD (PQ-MIP-RPAD) design was 


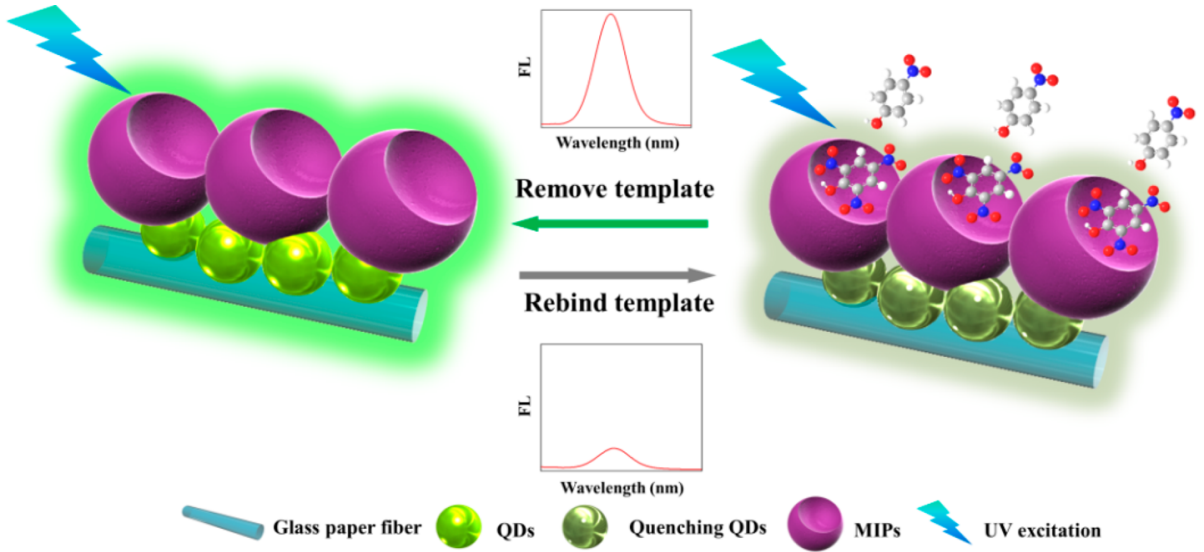

Figure 1. Schematic illustration of the structure and sensing principle of the rotational paper-based microfluidic chip (PQ-MIP RPAD).

implemented by being drawn in Adobe Illustrator software and then directly printed onto the filter paper (Whatman chromatography No. 1 paper, GE) by a wax printer (XEROX Phaser 8560DN) with a highest resolution of 2400 dpi (shown in Figure S1). Then, the wax-patterned paper was put into the oven at $120{ }^{\circ} \mathrm{C}$ for $120 \mathrm{~s}$. This process allowed the wax to penetrate through the whole paper completely and form hydrophobic barriers.

As shown in Figures S2A and S3, the rotational paper chip consists of three parts. The hexagonal bottom sensor plate has a length of $43 \mathrm{~mm}$ on each side and comprises six square $(7 \times$ $7 \mathrm{~mm}$ ) paper-based fluorescence sensors prepared in advance (PQ-4-NP-MIP and PQ-TNP-MIP). Three of these detect 4NP, and the other three sites detect TNP. The middle layer has a circular shape with a diameter of $60 \mathrm{~mm}$ and six square holes corresponding to the square paper-based fluorescence sensors of the bottom plate. The top sampling layer contains sample cells (diameters of $7 \mathrm{~mm}$ ) and hydrophilic channels corresponding to the middle layer.

The rotational-paper-chip assembling process is illustrated in Figures S2B and S3. Briefly, the bottom of the sensor plate was combined with the paper-based fluorescence sensors (PQ-4NP-MIP and PQ-TNP-MIP). Then, the top and middle layers were placed on the bottom sensing pad sequentially. Each sensing site was aligned to its corresponding square hole in the middle disk. Finally a hollow rivet was punched into the center hole of three paper discs with a hammer, which caused about 1.5 times expansion at the end of the hollow rivet. Finally, the three paper discs were assembled together and could be flexibly rotated.

\section{RESULTS AND DISCUSSION}

Fabrication and Operation of PQ-MIP RPADs and Detection Mechanism. The glass-fiber paper was modified with fluorescent quantum dots (QDs) on the surface and linked with a molecularly imprinted polymer layer. There were many impurities in the glass-fiber-paper surface, and it needed to be soaked at an $\mathrm{HCl}$ solution for a short time, which could not only remove impurities but also activate the silicon hydroxyl groups of the glass-fiber surfaces, thus benefiting the grafting of amino group to the glass fibers by APTES. The carboxyl groups on the surfaces of the CdTe QDs were connected to the amino groups on the surfaces of the glass fibers, generating amide bonds by using EDC-NHS catalysis to obtain a stable grafted quantum-dot substrate on glass fibers.
The phenolic hydroxyl groups of 4-NP and TNP were implemented via prepolymerization with the functional groups of APTES through the hydrogen bonds. Then, the template molecules (4-NP and TNP), cross-linker (tetraethyl orthosilicate, TEOS), and initiator $\left(\mathrm{NH}_{3} \cdot \mathrm{H}_{2} \mathrm{O}\right)$ were added to synthesize the imprinted polymers. Because of covalent bonds, the molecularly imprinted polymer grafted on the paper firmly, and the fluorescence signal of the wrapped quantum dots was kept stable. The sensing principle and constructed-paper-based-fluorescence-sensor structure are shown in Figure 1. Because of the existence of the template (4-NP and TNP), the fluorescence signal could be quenched and restored after the removal of the template by elution. A suitable synthesis process was the premise of the sensitivity and selectivity of the rotational paper chip.

Compared with the previously reported origami $\mu \mathrm{PAD}$, the rotational paper-based microfluidic chips provided a flexible and easy way to operate the entire process conveniently. Briefly, the entire rotational chip was composed of three layers (displayed in Figure 2A-C). Figure 2A shows a photograph of a PQ-MIP RPAD under normal light, and it can be pointed out that the size of the chip is small, which is beneficial for transportation. At the bottom layer, six hexagonal sensor plates are pasted (three sites for detecting 4-NP, and three sites for detecting TNP; Figure 2B). When UV light is emitted from the light source, it reaches the detection site of our chip, and the signal of 4-NP or TNP is recorded by the fluorescence spectrometer (Figure 2B,C). The center isolation disc has six square holes corresponding to the bottom sensor, which prevent cross-contamination of the sample from the fluid channel leaking into other sensing sites during rotation. The top layer contains the sample reservoir and the hydrophobic channel that facilitates the samples flowing along the channel to the sensor sites. Figure 2D shows the whole working process of the rotational chip. This rotational structure enables reliable, effective multiplexed analysis.

As shown in Figure 2D, the top layer was set with six round sample reservoirs connected to fluid channels that led to the corresponding sensing sites on the bottom layer for multiplexed analysis. During the test, $5 \mu \mathrm{L}$ samples were added to the sample reservoir and flowed along with the water channel to the MIP sensing area. After the reaction finished, we rotated the first layer of the RPAD $60^{\circ}$ to expose the sensing site and placed it on the holder of the instrument to detect the fluorescence signal. When the square hole of the top sample plate of the chip was aligned with the detection site, green 

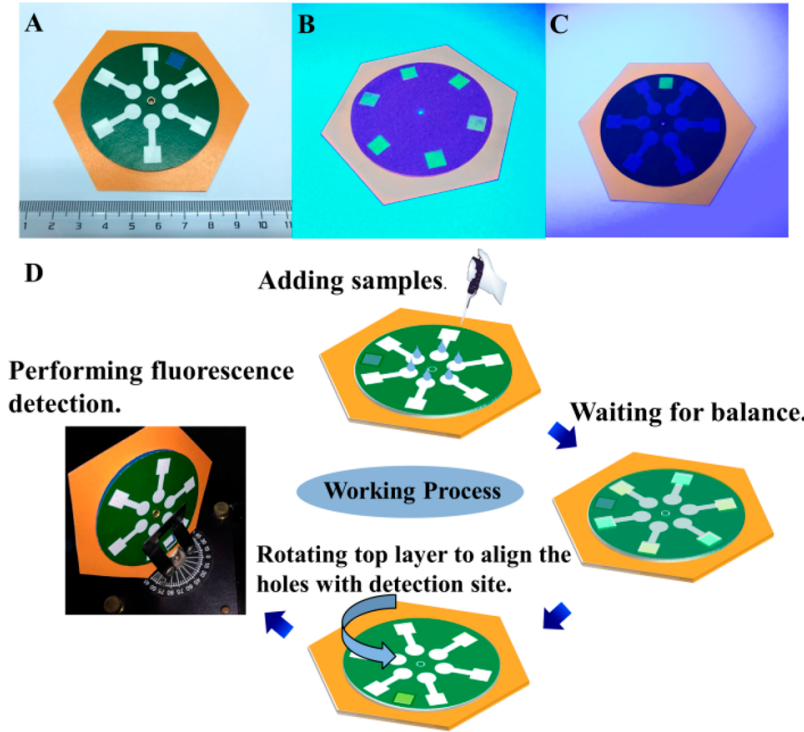

Figure 2. Illustrated use of the process and exhibition of the rotational paper-based microfluidic chips. (A) Appearance of a complete chip under daylight. (B) Photograph of the six test sites on the chip under UV light. (C) Photograph of the test site through the hole of the top sampling layer under UV light $(365 \mathrm{~nm})$. (D) Illustration of the whole working process of the rotational chip and a photograph of rotational paper-based microfluidic chips placed and the detection process in the fluorescence spectrometer. UV light: $396 \mathrm{~nm}$, slit: $5 \mathrm{~nm}$.

fluorescence could be seen. This flexible rotation enabled us to implement multiplexed detection on the chip. The surfaceimprinting technique makes the molecularly imprinted polymer and quantum-dot surface fit closely. Because of the hydrogen bonding between the phenolic hydroxyl groups and the molecular groups of the molecularly imprinted polymer, electron transfer occurs between the active phenolic hydroxyl groups and the quantum dots that quench the fluorescence. The effect of fluorescence quenching can be fitted by the Stem-Volmer equation:

$$
\left(F_{0} / F\right)-1=K_{\mathrm{SV}} C_{\mathrm{M}}(3.1)
$$

where $F_{0}$ represents the original fluorescence intensity of the imprinted fluorescent chip prior to the addition of the template molecule, $F$ is the fluorescence-intensity value after the addition of the template molecule (4-NP or TNP), $K_{\mathrm{SV}}$ is the quenching constant, and the concentration of the template molecule is represented by $C_{\mathrm{M}} . I_{\mathrm{F}}$ is the imprinting factor, which is the ratio of the $K_{S V}$ values of the PQ-MIP RPADs and the PQ-NIP RPADs, and $I_{F}$ is an important parameter that characterizes the selectivity of the PQ-MIP RPADs. This kind of surface-imprinting method can not only effectively protect quantum-dot fluorescence but also help to quickly identify and combine target molecules, which enhances the selectivity and mass-transfer efficiency of the system.

This indicated that the fluorescence signal of the sensing position chip of the PQ-MIP RPADs was excellent and convenient for fluorescence detection by a fluorescence spectrometer.

Characterization of PQ-MIP RPADs. The surface micromorphology of the paper-based FL-MIP sensors was characterized by cold-field-emission scanning electron microscopy (SEM) and fluorescence microscopy. Figure 3A shows the microstructure and high-power image of pure glass-fiber

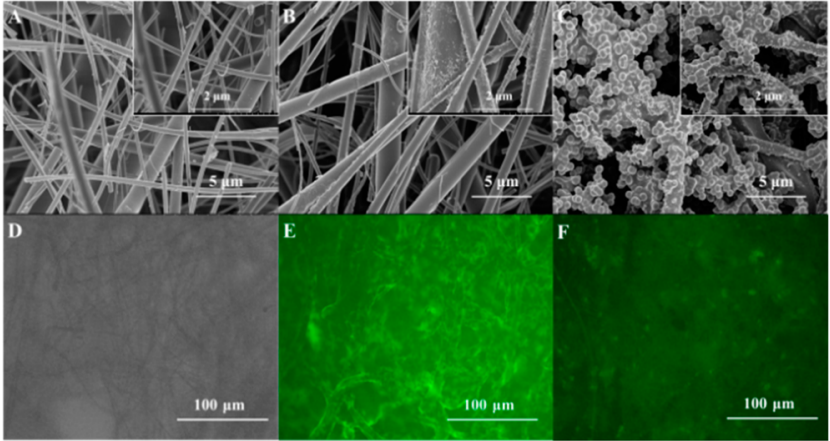

Figure 3. (A-C) Scanning-electron-microscopy images of (A) bare glass-fiber paper, (B) QD-modified glass-fiber paper, and (C) QDMIP-modified glass-fiber paper. Scale bars are 5 and $2 \mu \mathrm{m}$. (D-E) Fluorescence-microscopy images of (D) bare glass-fiber paper, (E) QD-modified glass-fiber paper, and (F) QD-MIP-modified glass-fiber paper. Scale bars are $100 \mu \mathrm{m}$.

paper. The picture shows an irregular, clear-dendritic-fiber structure with a fiber diameter of about $3-10 \mu \mathrm{m}$. Figure $3 \mathrm{~B}$ shows the paper chip grafted with quantum dots. It can be observed in the picture that the dendritic glass fibers have a thin and uniform layer of quantum dots. Because of the smaller size of the quantum dots, a larger layer of protrusions can be viewed at high magnification, which proves that the quantum dots were uniformly distributed on the glass fibers. Figure 3C shows that the surfaces of the glass fibers wrap a large number of spherical imprinted polymers, and high-magnification photos show that the diameter of the molecularly imprinted polymer sphere is about $0.2 \mu \mathrm{m}$. Figure $3 \mathrm{~A}-\mathrm{C}$ corresponds to the fluorescence-microscope photographs of Figure 3D-F. Figure $3 \mathrm{E}$ is a fluorescence-microscopy photograph of the chip after the grafting of the quantum dots. The figure shows relatively uniform and bright-green fluorescence emitting from the transparent layer of glass fibers, and the transparent glass fibers are visible in the figure. In Figure 3F, the fluorescence of the QDs has become dark because of the quenching of the QDs by the template molecule.

Optimization of Experimental and Practical Conditions of PQ-MIP RPADs. It is essential to optimize the conditions to improve the accuracy and stability of PQ-MIPRPAD detection. Under optimal conditions, this rotational structure would perform better for multiplexed detection and is highly compatible with fluorescence instruments. Therefore, we performed some comparative experiments to determine the optimal conditions.

First, modification of the surface of quantum dots was investigated, and quantum dots modified with TGA, L-Cys, and GSH were synthesized. As shown in Figure S4, it was evident that 4-nitrophenol (4-NP) had an excellent quenching effect on the glutathione-modified QDs for PQ-4-NP-MIP, probably because glutathione itself contains large amounts amino groups, and CdTe quantum dots exposed outside of the amino groups are more likely to form hydrogen bonds with 4$\mathrm{NP}$, which causes electron transfers that quench the fluorescence. After the measurements, the $\zeta$-potential values of thioglycolic acid doped CdTe QDs were between -30.1 and $-19.3 \mathrm{mV}$. Because 4-NP is acidic and positively charged in solution, high concentrations of 4-NP also cause fluorescence quenching of thioglycolic acid modified quantum dots by electron transfer. In order to amplify the amino groups possessed by functionalized monomers (APTES) in the 
molecularly imprinted polymer, thioglycolic acid modified CdTe QDs were used as fluorescent substrates for PQ-MIP RPADs.

It needs to be considered whether the sensitivity of the fluorescence response is affected by the dense distribution of $\mathrm{CdTe}$ quantum dots on the glass-fiber paper. After the design experiment, different amounts of CdTe QDs (5, 7.5, and 10 $\mathrm{mL}$ ) were modified on the glass-fiber paper. If the amount of the CdTe QDs was below $5 \mathrm{~mL}$, the fluorescence signal of the sensing part of the chip was too low to be detected. Taking 4$\mathrm{NP}$ as an example, the quenching effect of $5 \mathrm{~mL}$ of CdTe QDs on paper chips was significantly better than that using 7.5 or 10 $\mathrm{mL}$ of CdTe QDs. However, the fluorescence signal of the high 4-NP target concentration was easily saturated (50-100 mg/ L) with $5 \mathrm{~mL}$ of CdTe QDs grafted on the paper chip (Figure 4A). Hence, to obtain a better fluorescence response and a wider linear detection range, $7.5 \mathrm{~mL}$ of quantum dots was selected as the condition for the following experiments.
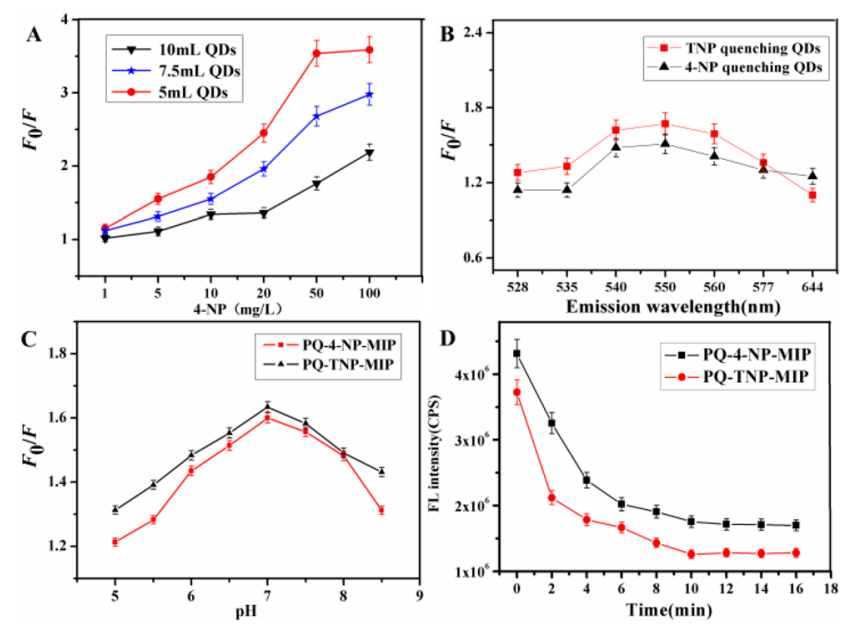

Figure 4. (A) Influence of the amount of quantum dots grafted on the quenching effects of PQ-MIP RPADs. (B) Influence of the different emission wavelengths of quantum dots on the quenching effects. (C) Influence of $\mathrm{pH}$ on the quenching effects of PQ-MIP RPADs. (D) Fluorescence intensities of PQ-MIP RPADs vs time within 16 min. The excitation wavelength and the emission wavelength were 396 and $550 \mathrm{~nm}$, respectively $(n=3)$.

Water-soluble CdTe quantum dots are easy to synthesize, and quantum dots with different emission wavelengths are synthesized by the different reaction heating times. The quenching effects of 4-NP and TNP on CdTe QDs with different emission wavelengths may be different because the wavelengths of $\mathrm{CdTe}$ quantum dots are related to the sizes of the CdTe quantum dots. We synthesized seven different kinds of CdTe QDs to fabricate molecularly imprinted paper chips with emission wavelengths of 528, 535, 540,550, 560, 577, and $644 \mathrm{~nm}$, respectively. As shown in Figure 4B, the quenching effect of CdTe QDs at 540-550 nm was better than that of QDs at other emission wavelengths. The quenching effect was relatively good, mainly because of the fact that the electron transfers between this size of quantum-dot particle and the target molecules 4-NP and TNP are strongest.

The effect of eluting the template after molecular imprinting was investigated by detecting the UV-visible spectrum of the eluent. The eluent was changed every $3 \mathrm{~min}$, and the UVvisible spectrum of the eluent was examined after a 5-fold dilution. As illustrated in Figure S5A,B, it was clearly observed that the template material was nearly eluted after $3 \mathrm{~min}$ according to its UV-vis-absorption peak. This implied that the template material could be detached from the molecularly imprinted polymers and form a cavity. To guarantee a good effect, we selected the condition of eluting twice for a total of $10 \mathrm{~min}$.

The optimization of the $\mathrm{pH}$ is shown in Figure 4C. The effect of fluorescence quenching by 4-NP was poor when the $\mathrm{pH}$ was less than 6 because the solution was too acidic and reduced the emission of the quantum dots. When the $\mathrm{pH}$ value increased from 6 to 7 , the fluorescence-quenching effect increased greatly, and the quenching effect reached its maximum at $\mathrm{pH}$ 7. The recognition sites on the surfaces of the imprinted polymers were protonated when the $\mathrm{pH}$ value was higher than 7.5, and the interactions between the molecular-imprinting-shell binding site and the template were reduced, which led to a sharp decrease of the fluorescencesignal-quenching effect. Taking into account the practical application of the sample analysis, phosphate-buffer solution $(0.01 \mathrm{~mol} / \mathrm{L}, \mathrm{pH} 7)$ was selected for further experimental conditions.

The binding-equilibrium times of the molecularly imprinted polymers with 4-NP and TNP were also evaluated. As displayed in Figure 4D, the decrease of the fluorescence signal was relatively rapid from the beginning of adsorption to $4 \mathrm{~min}$, and the fluorescence intensity decreased gently from 4 to 10 $\mathrm{min}$. After $12 \mathrm{~min}$, the fluorescence signal tended to be stable, and the equilibrium of adsorption could be determined. Therefore, we chose $15 \mathrm{~min}$ as the adsorption equilibrium time for further experiments and final applications.

Function of Detecting 4-NP and TNP on PQ-MIP RPADs. To verify the multiplexed simultaneous detection of environmental pollutants on RPADs, 4-NP and TNP were analyzed as the model analytes. From Figure 5A,B, we could see that the quenching-coefficient $\left(K_{\mathrm{SV}}\right)$ values of 4 -NP and TNP on the imprinted and nonimprinted chips were obviously different. The synthesized imprinted chip had better selectivity. The fluorescence-quenching effect of the imprinted RPADs was much larger than that of the nonimprinted RPADs in the presence of the same concentration of the template molecule. In addition, for the 4-NP imprinted chip (PQ-4-NP-MIP $\mathrm{RPAD}$ ), the fluorescence intensity of the imprinted chip decreased with increasing 4-NP concentrations when the 4-NP concentration was in the range of $0.5-20.0 \mathrm{mg} / \mathrm{L}$. The linear correlation coefficient, $R^{2}$, was 0.994 with a detection limit of $0.097 \mathrm{mg} / \mathrm{L}$. Meanwhile, the fluorescence intensity of the nonimprinted chip also decreased with increasing 4-NP concentrations, but the decrease was not obvious compared with that of the imprinted chip. The correlation coefficient, $R^{2}$, was 0.957 . The calculation of the quenching constant indicated that there were a large number of recognition sites on the imprinted chip PQ-4-NP-MIP RPAD. As shown in Figure 5C,D, when the concentration of TNP was $0.5-20.0 \mathrm{mg} / \mathrm{L}$, the change in the fluorescence intensity was linear. The correlation coefficient, $R^{2}$, was 0.991 , and the detection limit was $0.071 \mathrm{mg} / \mathrm{L}$. At the same time, the fluorescence intensity of the nonimprinted chip also decreased with increasing TNP concentrations, but the decrease was not obvious compared with that of the imprinted chip. The correlation coefficient, $R^{2}$, was 0.997.

2-NP, 2-aminophenol (PAP), hydroquinone (HQ), and bisphenol A (BPA) were selected as the interferences to 

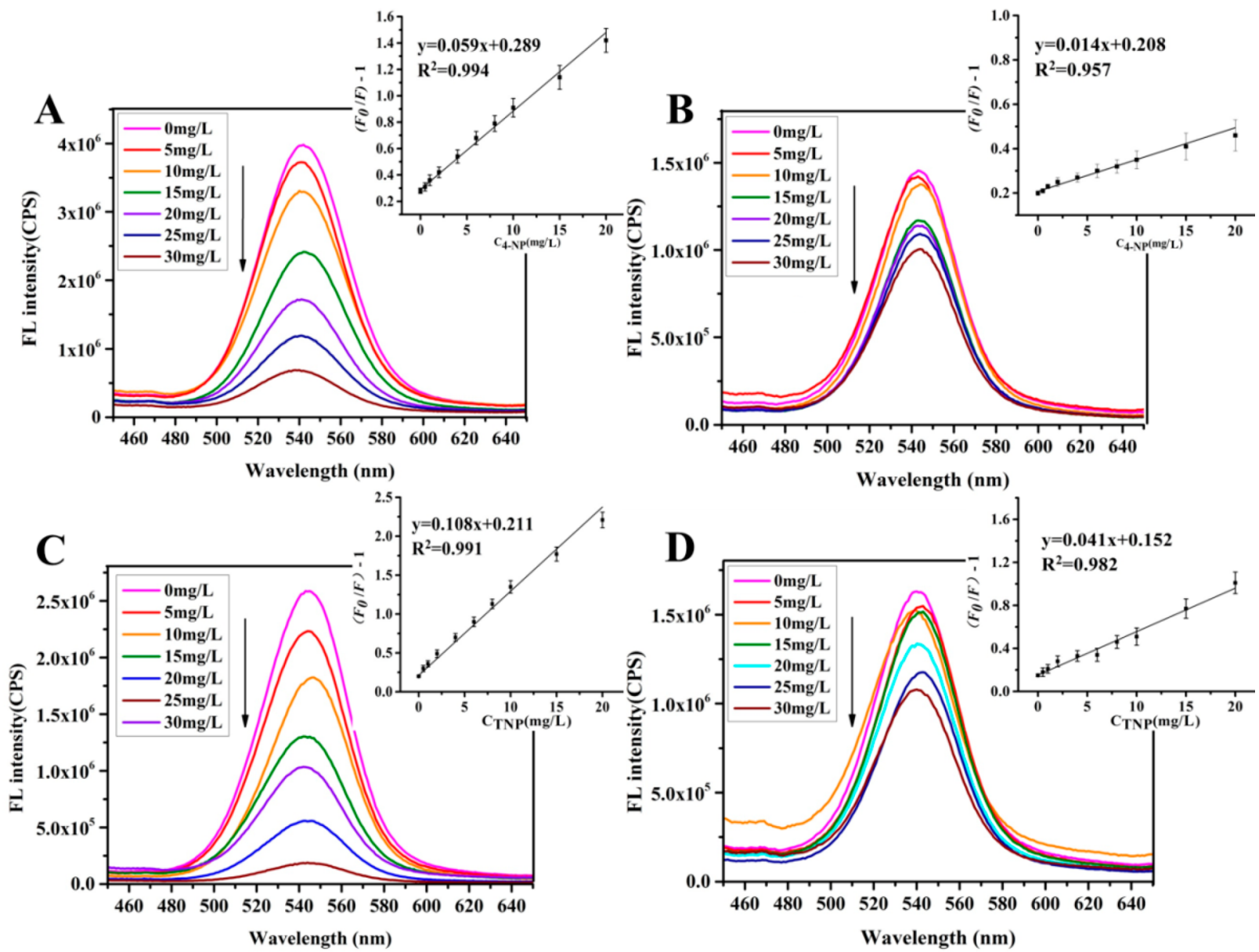

Figure 5. Quenching effects in the fluorescence spectra of (A) PQ-4-NP-MIP RPADs, (B) PQ-4-NP-NIP RPADs, (C) PQ-TNP-MIP RPADs, and (D) PQ-TNP-NIP RPADs. The concentrations of 4-NP and TNP were 0, 5, 10, 15, 20, 25, and $30 \mathrm{mg} / \mathrm{L}$. The corresponding Stern-Volmer-plot curves of the PQ-MIP and PQ-NIP RPADs are shown in the insets. The experimental conditions were $\mathrm{pH} 7.0$ and room temperature; the excitation wavelength was $396 \mathrm{~nm}$, and the slit widths of emission and excitation were $5 \mathrm{~nm}(n=3)$.

investigate the specific adsorption capacities of the 4-NP-MIP RPADs and PQ-TNP-MIP RPADs. The adsorption time was $15 \mathrm{~min}$ and the concentration of each component was $10 \mathrm{mg} /$ L. The results are shown in Figure 6A,B. The corresponding results of the fluorescence quenching of the PQ-MIP RPADs with 4-NP and TNP were the highest, followed by the fluorescence-quenching effects of 2-NP and PAP, which were larger than those of HQ and PBA. The HQ and PBA effects were very similar and relatively low. The specific-recognition
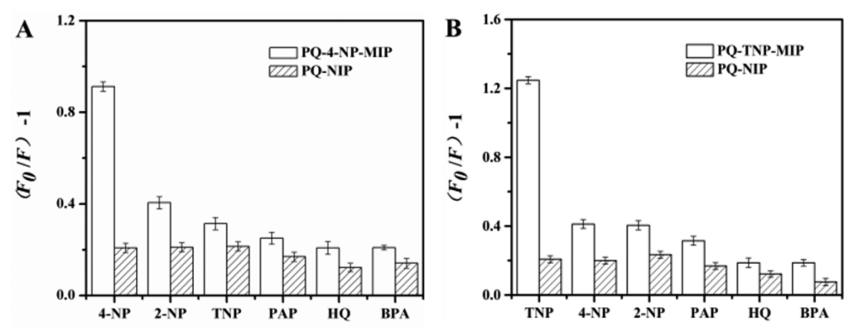

Figure 6. Selectivity of (A) PQ-4-NP-MIP RPADs and PQ-NIP RPADs and (B) PQ-TNP-MIP RPADs and PQ-NIP RPADs for sample solutions of different interfering molecules (2-NP, 2aminophenol (PAP), hydroquinone (HQ), and bisphenol A $(\mathrm{BPA}))$. The concentrations of 4-NP, TNP, and the interfering molecules were $10 \mathrm{mg} / \mathrm{L}(n=3)$. effect of the molecularly imprinted polymers using 4-NP and TNP as templates was well reflected in the synthesis process because the imprinted material had a large number of holes that specifically recognized 4-NP and TNP, and thus 4-NP and TNP could provide strong fluorescence-quenching effects at the sensing sites where the molecularly imprinted polymers were synthesized. The spatial configuration of $2-\mathrm{NP}$ is different from that of 4-NP, but its molecular composition and molecular size are similar, and the phenolic hydroxyl group on 2-NP is quite active. Therefore, some 2-NP entered the 4NP cavity, resulting in a certain degree of fluorescence quenching. The interference abilities of 4-NP and 2-NP were basically the same. The intricate structure of the glass fibers causes uneven coverage of the imprinted material, and the quantum dots exposed on the outside are quenched by the interference. The molecular weights of aminophenol and bisphenol A differ greatly from those of 4-NP and TNP, and their phenolic hydroxyl groups are not as active as those of 4NP and TNP. Therefore, they cannot be recognized by the cavities of the molecularly imprinted materials, but they were still partially exposed to quantum dots, causing weaker quenching. The result of the selectivity study showed that the common analogues do not cause any fatal interference with the test results. PQ-4-NP-MIP RPADs and PQ-TNP-MIP RPADs have better abilities to specifically recognize 4-NP and TNP, which can be better applied in practice. 
When we used the paper@QDs@4-NP-MIP sites, it was worth considering whether there was any variability in the fabrication process. Herein, the experiments were designed to implement fluorescence detection for 15 different paper@ QDs@4-NP-MIP sites, as shown in Figure S6, and there were no obvious differences among the results (RSD of $3.28 \%$ ). We also investigated the storage stability of the fluorescence signal of the chip over 15 days (Figure S7), and the results demonstrated good storage stability.

Through successful analysis of 4-NP and TNP experiments, $\mathrm{RPAD}$ clearly demonstrates excellent performance in multiplexed simultaneous detection and is compatible with fluorescence instrumentation.

Practical Application of PQ-MIP RPADs. In order to test the practical application of the PQ-MIP RPADs, we assembled 4-NP imprinted fluorescent chips and TNP imprinted fluorescent chips on RPADs to detect 4-NP and TNP simultaneously in six groups of samples. Lake-water samples were obtained from Panchi Lake of Shanghai University, and the seawater samples were taken from the coastal zone of the Yellow Sea of China. The 4-NP and TNP spiked concentrations were 5,10 , and $15 \mathrm{mg} / \mathrm{L}$. Table 1 shows the

Table 1. Recovery of Spiked 4-NP and TNP in Lake Water and Seawater Detected Using PQ-MIP RPADs $(n=5)$

\begin{tabular}{|c|c|c|c|c|c|}
\hline \multicolumn{2}{|c|}{ added } & \multicolumn{2}{|c|}{ found } & \multicolumn{2}{|c|}{ recovery $\pm \mathrm{RSD}$} \\
\hline $\begin{array}{c}\text { 4-NP } \\
(\mathrm{mg} / \mathrm{L})\end{array}$ & $\begin{array}{c}\mathrm{TNP} \\
(\mathrm{mg} / \mathrm{L})\end{array}$ & $\begin{array}{c}\text { 4-NP } \\
(\mathrm{mg} / \mathrm{L})\end{array}$ & $\begin{array}{c}\mathrm{TNP} \\
(\mathrm{mg} / \mathrm{L})\end{array}$ & 4-NP (\%) & TNP (\%) \\
\hline \multicolumn{6}{|c|}{ Lake Water } \\
\hline 0 & 0 & 0 & 0 & - & - \\
\hline 5 & 5 & 4.98 & 5.04 & $99.6 \pm 4.7$ & $100.8 \pm 4.2$ \\
\hline 10 & 10 & 9.63 & 9.26 & $96.3 \pm 4.8$ & $92.6 \pm 4.1$ \\
\hline 15 & 15 & 15.16 & 14.67 & $101.1 \pm 5.5$ & $97.8 \pm 4.3$ \\
\hline \multicolumn{6}{|c|}{ Sea Water } \\
\hline 0 & 0 & 0 & 0 & - & - \\
\hline 5 & 5 & 5.25 & 5.33 & $105.1 \pm 4.7$ & $106.6 \pm 4.3$ \\
\hline 10 & 10 & 9.67 & 9.51 & $96.7 \pm 4.6$ & $95.1 \pm 4.1$ \\
\hline 15 & 15 & 14.82 & 14.96 & $98.8 \pm 4.1$ & $99.7 \pm 3.2$ \\
\hline
\end{tabular}

recoveries from the lake-water and seawater samples ranging from 92.6 to $106.6 \%$ with RSDs ranging from 3.2 to $5.5 \%$. As shown in Figure S8A,B, for the actual samples, the designed sample channel had a good filtering effect. To prove this, we chose three kinds of water, including muddy water from a shallow area, sandy seawater, and algae-rich lake water. It can be seen that the dirty contaminants can be filtered out very well from the unclean water samples. We observed that the 5 $\mu \mathrm{L}$ samples had the ability to flow through the water channel to the MIP sensing area (Figure S8C). The results of real samples show that PQ-4-NP-MIP RPADs and PQ-TNP-MIP RPADs can be used to detect 4-NP and TNP simultaneously in complex environmental water samples with high accuracy and that they have a great potential for practical use.

Comparison of the Different Methods' Performances. Most recently reported analysis methods use electron transfer and $\pi-\pi$ interactions to quench the fluorescence signals of fluorescent substances and achieve detection, as shown in Table S1. ${ }^{36-42}$ Molecularly imprinted systems show better selectivity and sensitivity. Some methods are very sensitive, but it is difficult to guarantee selectivity and easy to have interference from the same species. Our PQ-MIP RPADs overcame this defect and allowed the simultaneous detection of two phenolic contaminants (TNP and 4-NP) in a multiplexed way.

\section{CONCLUSION}

In this work, we demonstrate a simple, versatile, and easy-touse rotational-PAD platform for detecting environmental pollutants. To the best of our knowledge, this is the first demonstration to combine fluorescence detection with the molecular-imprinting technique on rotational PADs. To demonstrate the broad applicability of this method, two phenolic pollutants (4-NP and TNP), as model targets, were determined by the proposed method. In addition, compared with the previously reported $3 \mathrm{D}$ origami $\mu$ PADs, this kind of rotational $\mathrm{PAD}$ showed some significant prospects: (1) The $360^{\circ}$ rotating structure enabled the paper-based chip to increase the utilization of chip's space and to achieve multiplexed detection. (2) The rotational microfluidic-chip design was ingenious, and the three-in-one nonseparable chip structure was flexible and effective, avoiding the folding or pressing of paper materials and easily achieving a multistep testing process. Moreover, the method showed good performance and advantages, such as a low cost, high selectivity and sensitivity, and excellent stability. It is expected that this reliable PQ-MIP RPAD platform will have enormous potential and widespread application prospects in environmental testing, food safety, and on-site detection.

\section{ASSOCIATED CONTENT}

\section{S Supporting Information}

The Supporting Information is available free of charge on the ACS Publications website at DOI: 10.1021/acs.analchem.8b01291.

More experimental materials and details, synthesis steps, and compound characterization (PDF)

\section{AUTHOR INFORMATION}

\section{Corresponding Authors}

*E-mail: bwli@yic.ac.cn (B.L.).

*E-mail: luck@shu.edu.cn (L.L.).

*E-mail: 1xchen@yic.ac.cn (L.C.).

ORCID

Bowei Li: 0000-0001-6262-8248

Lingxin Chen: 0000-0002-3764-3515

Notes

The authors declare no competing financial interest.

\section{ACKNOWLEDGMENTS}

This work was financially supported by the National Natural Science Foundation of China (Grant Nos. 41776110, 21804010, 61571278, 61571280, and 21876199), the National Key Research and Development Program of China (Grant No. 2016YFC1400702), and the Science and Technology Development Plan of Yantai (Grant Nos. 2015ZH087 and 2017ZH089).

\section{REFERENCES}

(1) Martinez, A. W.; Phillips, S. T.; Whitesides, G. M.; Carrilho, E. Anal. Chem. 2010, 82, 3-10.

(2) Li, B.; Zhang, W.; Chen, L.; Lin, B. Electrophoresis 2013, 34, $2162-2168$.

(3) Li, B.; Yu, L.; Qi, J.; Fu, L.; Zhang, P.; Chen, L. Anal. Chem. 2017, 89, 5707-5712. 
(4) Liu, H.; Crooks, R. M. J. Am. Chem. Soc. 2011, 133, 1756417566.

(5) Tian, T.; An, Y.; Wu, Y.; Song, Y.; Zhu, Z.; Yang, C. ACS Appl. Mater. Interfaces 2017, 9, 30480-30487.

(6) López-Marzo, A. M.; Merkoçi, A. Lab Chip 2016, 16, 31503176.

(7) Teengam, P.; Siangproh, W.; Tuantranont, A.; Vilaivan, T.; Chailapakul, O.; Henry, C. S. Anal. Chem. 2017, 89, 5428-5435.

(8) Figueredo, F.; Garcia, P. T.; Cortón, E.; Coltro, W. K. ACS Appl. Mater. Interfaces 2016, 8, 11-15.

(9) Yu, J.; Ge, L.; Huang, J.; Wang, S.; Ge, S. Lab Chip 2011, 11, $1286-1291$.

(10) Glavan, A. C.; Christodouleas, D. C.; Mosadegh, B.; Yu, H. D.; Smith, B. S.; Lessing, J.; Fernández-Abedul, M. T.; Whitesides, G. M. Anal. Chem. 2014, 86, 11999-12007.

(11) Sun, X.; Li, B.; Tian, C.; Yu, F.; Zhou, N.; Zhan, Y.; Chen, L. Anal. Chim. Acta 2018, 1007, 33-39.

(12) Qi, J.; Li, B.; Wang, X.; Zhang, Z.; Wang, Z.; Han, J.; Chen, L. Sens. Actuators, $B$ 2017, 251, 224-233.

(13) Sun, H.; Li, W.; Dong, Z. Z.; Hu, C.; Leung, C. H.; Ma, D. L.; Ren, K. Biosens. Bioelectron. 2018, 99, 361-367.

(14) Fiedoruk-pogrebniak, M.; Granica, M.; Koncki, R. Talanta 2018, 178, 31-36.

(15) Guzman, J. M. C. C.; Tayo, L. L.; Liu, C. C.; Wang, Y. N.; Fu, L. M. Sens. Actuators, B 2018, 255, 3623-3629.

(16) Zang, D.; Ge, L.; Yan, M.; Song, X.; Yu, J. Chem. Commun. 2012, 48, 4683-4685.

(17) Martinez, A. W.; Phillips, S. T.; Whitesides, G. M. P. Proc. Natl. Acad. Sci. U. S. A. 2008, 105, 19606-19611.

(18) Shin, J. H.; Park, J. K. Anal. Chem. 2016, 88, 10374-10378.

(19) Gong, M. M.; Sinton, D. Chem. Rev. 2017, 117, 8447-8480.

(20) Wouters, S.; De Vos, J.; Dores-Sousa, J. L.; Wouters, B.; Desmet, G.; Eeltink, S. J. Chromatogr. A 2017, 1523, 224-233.

(21) Yamada, K.; Henares, T. G.; Suzuki, K.; Citterio, D. ACS Appl. Mater. Interfaces 2015, 7, 24864-24875.

(22) Ju, K. S.; Parales, R. E. Microbiol. Mol. Biol. R. 2010, 74, 250272.

(23) Masqué, N.; Marcé, R. M.; Borrull, F.; Cormack, P. A. G.; Sherrington, D. C. Anal. Chem. 2000, 72, 4122-4126.

(24) Nagarkar, S. S.; Desai, A. V.; Ghosh, S. K. CrystEngComm 2016, $18,2994-3007$.

(25) Alkasir, R. S.; Ornatska, M.; Andreescu, S. Anal. Chem. 2012, 84, 9729-9737.

(26) Sun, G.; Wang, P.; Ge, S.; Ge, L.; Yu, J.; Yan, M. Biosens. Bioelectron. 2014, 56, 97-103.

(27) Kong, Q.; Wang, Y.; Zhang, L.; Ge, S.; Yu, J. Sens. Actuators, B 2017, 243, 130-136.

(28) Liu, J. X.; Chen, H.; Lin, Z.; Lin, J. M. Anal. Chem. 2010, 82, $7380-7386$.

(29) Wang, X.; Yu, J.; Kang, Q.; Shen, D.; Li, J.; Chen, L. Biosens. Bioelectron. 2016, 77, 624-630.

(30) Wang, H. F.; He, Y.; Ji, T. R.; Yan, X. P. Anal. Chem. 2009, 81, $1615-1621$.

(31) Chen, L.; Wang, X.; Lu, W.; Wu, X.; Li, J. Chem. Soc. Rev. 2016, 45, 2137-2211.

(32) Chen, L.; Xu, S.; Li, J. Chem. Soc. Rev. 2011, 40, 2922-2942.

(33) Xu, S.; Lu, H.; Li, J.; Song, X.; Wang, A.; Chen, L.; Han, S. ACS Appl. Mater. Interfaces 2013, 5, 8146-8154.

(34) Wei, X.; Hao, T.; Xu, Y.; Lu, K.; Li, H.; Yan, Y.; Zhou, Z. Sens. Actuators, B 2016, 224, 315-324.

(35) Li, B.; Zhang, Z.; Qi, J.; Zhou, N.; Qin, S.; Choo, J.; Chen, L. ACS Sensors 2017, 2, 243-250.

(36) Li, D. Y.; He, X. W.; Chen, Y.; Li, W. Y.; Zhang, Y. K. ACS Appl. Mater. Interfaces 2013, 5, 12609-12616.

(37) Hao, T.; Wei, X.; Nie, Y.; Xu, Y.; Yan, Y.; Zhou, Z. Microchim. Acta 2016, 183, 2197-2203.

(38) Li, W.; Zhang, H.; Chen, S.; Liu, Y.; Zhuang, J.; Lei, B. Biosens. Bioelectron. 2016, 86, 706-713.
(39) Liu, J. X.; Chen, H.; Lin, Z.; Lin, J. M. Anal. Chem. 2010, 82, $7380-7386$

(40) Han, Y.; Chen, Y.; Feng, J.; Liu, J.; Ma, S.; Chen, X. Anal. Chem. 2017, 89, 3001-3008.

(41) Kumar, A.; Chae, P. S. Sens. Actuators, B 2017, 240, 1-9.

(42) Peng, Y.; Zhang, A. J.; Dong, M.; Wang, Y. W. Chem. Commun. 2011, 47, 4505-4507. 